\title{
Comunicación plurilingüe en clases de francés de turismo: implementación del enfoque accional
}

Jean Noel Cooman

Escuela de Lenguas y Culturas Extranjeras

Universidad Nacional Autónoma de Honduras

\section{Resumen}

En este artículo se analiza de qué manera se puede desarrollar con un grupo de estudiantes universitarios principiantes de francés, una competencia plurilingüe y pluricultural $^{1}$ y de manera simultánea. Se presentan los resultados de una intervención educativa cuyo propósito fue aplicar el enfoque comunicativo accional dentro del aula, observando dos estudiantes durante un proceso de (semi)auto-aprendizaje, dirigido por un equipo de dos profesores trabajando en binomio y durante un intercambio oral con un grupo de turistas franceses e ingleses invitados al aula.

Con miras a la realización de esta conversación trilingüe (francés-inglés-español), que consistía en presentar a los turistas un programa atractivo de actividades turísticas en Honduras, los estudiantes se entrenaron no solamente para construir discursos auténticos en tres lenguas, sino también se prepararon para construir su competencia pluricultural a través de actividades de reflexión cultural e intercultural.

A través del análisis de los discursos producidos, se comprobó que los dos estudiantes experimentales fueron capaces de construir su repertorio plurilingüe y pluricultural de manera diferente pero funcionalmente aceptable, logrando precisamente efectuar una comunicación exolingüe ${ }^{2}$ con sus interlocutores extranjeros, durante la cual recurrieron a estrategias plurilingües caracterizadas por la creación de articula- ciones originales entre las lenguas en cuestión.

En base a los resultados de dicho experimento se pretende formular además, propuestas de creación de actividades comunicativas en varias lenguas, basadas en el enfoque accional, y aplicable en el contexto educativo hondureño.

Palabras clave: clase plurilingüe, repertorio plurilingüe, repertorio pluricultural, comunicación plurilingüe, comunicación exolingue, auto-aprendizaje.

1 La competencia para comunicar lingüísticamente e interactuar culturalmente -desarrollando también una competencia intercultural-, de un aprendiente -actor social- que domina a niveles diferentes, diferentes lenguas y culturas (Coste, Moore y Zarate, 1998).

2 Toda interacción verbal de "cara a cara", caracterizada por divergencias particulares significativas entre los repertorios lingüísticos de los participantes (Porquier, 1984). 


\section{Introducción}

Aunque Honduras es oficialmente un país multilingüe y multicultural, al igual que la mayoría de sus vecinos latinoamericanos está marcado por una presencia muy fuerte del español, que compromete considerablemente la existencia de las otras lenguas vernáculas y minoritarias del país. Esta posición dominante del castellano se ha reflejado además, en la instauración en el sistema público de una sola lengua extranjera obligatoria el inglés, que ha podido reforzar su presencia en virtud de la fuerte influencia económica de los Estados Unidos.

Por su parte, el francés ha logrado conquistar su espacio como segunda lengua extranjera y se enseña en el país desde hace mucho tiempo, no solamente en las dos grandes universidades públicas sino también en otras universidades y en escuelas bilingües de las ciudades de Tegucigalpa y San Pedro Sula; y como segunda lengua optativa en el sistema público a la par de su hermano mayor, el inglés.

La coexistencia de estas lenguas extranjeras a todo nivel educativo explica en parte la realización, en un contexto de enseñanza-aprendizaje del francés en la Universidad Nacional Autónoma de Honduras (UNAH), de una experiencia de clase que se presenta a continuación. A través de este experimento se analizó la construcción de un repertorio verbal plurilingüe y pluricultural, caracterizado precisamente por el desarrollo de una competencia en francés que se nutre de una competencia ya existente en español y en inglés.

Esta construcción está inscrita dentro de un enfoque "plural" de aprendizaje de lenguas, cuyo carácter innovador se diferencia precisamente del aprendizaje tradicional, en que en este se aprenden las "nuevas" lenguas una después de otra, y sin establecer conexiones entre dichos aprendizajes.

En cuanto a la metodología utilizada, el proyecto de clase se llevó a cabo a través de un enfoque accional desarrollado en el Marco Común Europeo de Referencia (2001), y que implica la puesta en práctica de tareas de aprendizaje que soliciten una ejecución, de un producto colectivo, construido mediante la colaboración y la negociación entre los estudiantes en el aula (Borg, 2001); y si posible mediante la interacción con interlocutores diversos fuera de ella.

Esta perspectiva accional pretende enriquecer más la metodología tradicional comunicativa que centraba el aprendizaje en la mera comunicación con el interlocutor, sobre todo el profesor y/o los otros aprendientes, y de manera casi exclusiva dentro del aula. En este proceso de cons- trucción del repertorio plurilingüe y pluricultural se describen, en primer lugar, las modalidades de implementación del proyecto, que forma parte de un experimento con dos estudiantes observados de cerca, y la realización precisa de sus competencias plurilingües y pluriculturales.

Posteriormente se examinan las modalidades específicas de aprendizaje-enseñanza de estas dos competencias plurales, para analizar finalmente su resultado a través del funcionamiento y características específicas, tal como quedaron reflejadas en los discursos finales de los estudiantes.

\section{Metodología de \\ desarrollo}

\section{Realización de la experien- cia de clase}

El proyecto de clase multilingüe se llevó a cabo con un público bastante homogéneo: 20 estudiantes hondureños con edades comprendidas entre 18 y 27 años, cursando la clase de Francés I, en la Escuela de Lenguas y Culturas Extranjeras de la UNAH, la cual tiene una duración de 60 horas a lo largo del período. El objetivo principal consistió en comprender las condiciones para la construcción de un repertorio plurilingüe y pluricultural en un grupo de estudiantes principiantes. De manera complementaria, se trataba de demostrar cómo el enfoque 
accional, aplicado en el campo de la enseñanza del francés, por objetivos específicos en el área de turismo, puede ser operativo en un contexto hondureño, y cómo una metodología de trabajo basada en el trabajo pedagógico "en binomio" y en una autonomía de aprendizaje, puede ser una herramienta útil para la construcción de competencias plurilingües $y$ pluriculturales.

El experimento consistió, concretamente, en formar a estos veinte estudiantes para asumir el papel de un guía de agencia de viajes, que explica en francés un breve programa de actividades turísticas a un grupo de turistas francófonos recién llegados al país, y que responde en español a las intervenciones de los turistas. Luego, de manera casi simultánea, un subgrupo de diez estudiantes voluntarios, llamados los "tri", fueron formados para explicar el mismo programa en idioma inglés a turistas ingleses, respondiendo también en español a las intervenciones de los turistas, utilizando de esta manera tres lenguas. De este subgrupo, fueron seleccionados y observados de cerca dos estudiantes, uno de ingeniería civil y uno de medicina , uno del sexo femenino y otro masculino: el primero, un estudiante de buen desempeño (de buena disposición, apertura de espíritu y de buenos resultados en la clase), y la segunda, una estudiante más atrás, (un tanto "bloqueada" y de "rendimiento regular e incluso bajo" y de resultados más bajos en el aula).

En primer lugar, la preparación abordó el desarrollo de la competencia "plurilingüe", tal como la definen Coste, Moore, y Zarate (1998), para proporcionar una formación trilingüe en las habilidades de comprensión oral (CO), de expresión oral (EO) y en menor medida en comprensión escrita (CE); también se pretendió desarrollar la competencia "pluricultural" (ibíd.), que consistía en hacer superar los estereotipos culturales en las tres culturas involucradas: la francesa, la inglesa y la hondureña. Todo esto habría de ayudar a asumir mejor el papel de guía en un contexto hondureño.

\section{Etapa 1: Preparación y rea-} lización del intercambio trilingüe

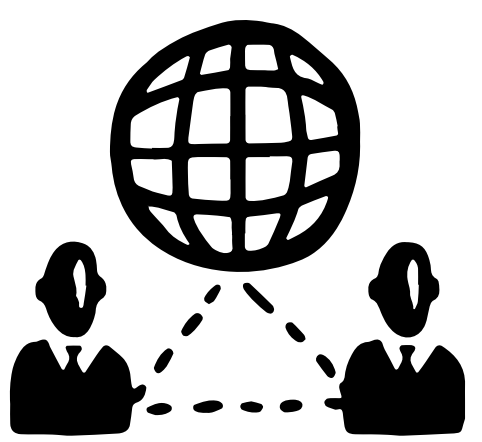

Para el desarrollo de la competencia plurilingüe, se diseñaron dos fases: una de "preparación y entrenamiento" y otra de "puesta en marcha", en cumplimiento de uno de los principios básicos del manual de formación Vacaciones en América Central (VAG), ex- puesto por Gremmo (1997); según el cual el aprendizaje de la comunicación se desarrolla respetando las tres fases consecutivas: "prepararse, entrenarse y lanzarse." Esta fase final, consistió precisamente en realizar el intercambio trilingüe.

Durante estas dos fases, los estudiantes se prepararon siguiendo en un primer momento los discursos modelo de las actividades del manual VAC, apropiándose de los instrumentos lingüísticos y discursivos que les permitieron realizar la explicación del programa turístico en tres lenguas, como guía para una agencia de viajes. Concretamente, las actividades de $\mathrm{CO}$ les prepararon para la comprensión de los distintos tipos de intervenciones de los turistas, (preguntas, peticiones, entre otros), mientras que las actividades de EO les permitieron concretizar la explicación del programa en tres lenguas.

En un segundo momento, durante la sub-fase de preparación directa a la realización del intercambio con los turistas, los estudiantes fueron entrenados para construir, en auto aprendizaje, los discursos auténticos de explicación de un lugar turístico de Honduras, desarrollados en francés. Para tal fin diseñaron esquemas escritos de su presentación y memorizaron la conversación. En cuanto al grupo de los estudiantes "tri" y los dos estudiantes observados, estos hicieron el trabajo en 
inglés, casi simultáneamente al reutilizar sus conocimientos y habilidades previamente adquiridas en las actividades de $\mathrm{CO}$ y $\mathrm{EO}$ en el otro idioma, con la misma metodología, y con ayuda de los mismos discursos traducidos al inglés. Además, conviene precisar que estas actividades de preparación fueron acompañadas de un trabajo de documentación-investigación sobre los lugares turísticos en Honduras, susceptibles de despertar el interés de turistas franceses e ingleses.

\section{Etapa 2: Preparación al con- tacto intercultural}

De forma paralela y complementaria a las actividades lingüístico-comunicativas, se desarrollaron sesiones de debate, con el fin de construir la competencia pluricultural e identitaria, tomando como referencia un diagnóstico realizado al inicio del curso, en el cual se identificaban las representaciones estereotipadas iniciales sobre Francia y los franceses, Inglaterra y los ingleses por una parte y Honduras y los hondureños por otra.

El trabajo posterior de evaluación de la pertinencia de estas representaciones, a través del análisis de su modo de construcción e internalización fue completado con una actividad de cotejo de estas representaciones, en una entrevista preliminar de los estudiantes con los turistas, invitados previamente al aula. La entrevista abordó todo tipo de aspectos culturales de sus respectivos países.

El objetivo era poder apreciar después de la conversación, la evolución de las representaciones de los estudiantes sobre estos países y sus habitantes, de manera comparativa con Honduras y los hondureños.

Por otra parte, las actividades de reflexión cultural e intercultural como las propuestas en el manual VAC, fueron llevadas a cabo, con el objetivo de comprender mejor el papel de guía; es decir, sus conocimientos, habilidades y actitudes específicas.

Finalmente, los estudiantes pusieron en práctica sus competencias plurilingües y pluriculturales, mediante la actividad de conversación bi y trilingüe, desempeñando el papel de guía y "embajador" de su patrimonio turístico frente a los turistas franceses e ingleses invitados. Concretamente, cuatro recorridos atractivos de Honduras fueron expuestos por cuatro grupos de estudiantes, dos de los cuales eran de los "tri", en donde se encontraban los dos estudiantes observados. Estos, después de haber presentado su programa de dos días en francés a los turistas franceses, siguieron con su presentación en inglés, concluyendo las dos presentaciones con respuestas en español a las intervenciones de los turistas.

\section{Proceso para la adquisición de habilidades plurilingües y pluriculturales: enseñanza en binomio y aprendizaje en autonomía}

Para orientar mejor el desarrollo de la competencia plurilingüe y pluricultural, específicamente en relación con la integración de dos lenguas y culturas extranjeras de forma simultánea, y para garantizar una adquisición más coordinada de los dos tipos de competencia, se consideró necesario introducir las siguientes modalidades de enseñanza-aprendizaje:

\section{El trabajo coordinado en equipo}

Mediante esta modalidad de trabajo en binomio, es decir entre dos profesores que compartieron el mismo espacio académico, se trató de organizar sesiones de clase en paralelo, incluso tripartitas. De esta manera, mientras que la primera parte del curso se desarrollaba en francés, en la segunda parte, bajo la modalidad de binomio, un maestro se hacía cargo del grupo "bi", organizando una sesión de reforzamiento de una actividad de $\mathrm{CO}$ o de $\mathrm{EO}$ ya realizada en la primera parte en francés; mientras que el otro colega desarrollaba la actividad equivalente en inglés, con el grupo "tri".

\section{El aprendizaje en autono- mía \\ Un enfoque más sistemático de auto-aprendizaje se intro-}




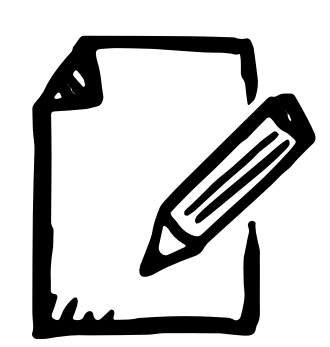

dujo en los estudiantes a través de las actividades siguientes:

a) La realización sistemática de un trabajo de auto-evaluación y de co-evaluación de su desempeño en $\mathrm{CO}$ y EO; por ejemplo, a través de la grabación de sus propios discursos, una herramienta considerada como un medio por excelencia para estimular la escucha y evaluación de sus propias producciones orales.

b) La lectura dirigida y reflexión sobre los "Consejos para aprender" propuestos en el manual $\mathrm{VAC}$, a fin de establecer las estrategias que pueden resultar más adecuadas para desarrollar las distintas competencias de manera individual.

c) La aplicación de encuestas. Por una parte, se aplicó un cuestionario-diagnóstico para medir las representaciones iniciales de todos los estudiantes participantes, respecto del aprendizaje y comunicación plurilingüe y un cuestionario final de auto-evaluación del desempeño plurilingüe; así como dos entrevistas semi directivas a los estudiantes "tri", que tenían como objetivo dirigir el aprendizaje plurilingüe y pluricultural.
Por otra parte, se establecieron tres cuestionarios en forma de diario de evaluación, dirigidos a todos los estudiantes y concebidos no solamente como instrumentos que permitieron medir la evolución en sus representaciones, sino también como biografía lingüística para evidenciar los recorridos de experiencia plurales de los estudiantes, tanto a nivel lingüístico como cultural. Finalmente, estos diarios contribuyeron a instaurar una mayor conciencia reflexiva acerca de las estrategias de aprendizaje plurilingües y pluriculturales.

\section{Análisis del discurso plu- rilingüe}

En cuanto a la realización concreta de la competencia plurilingüe en la interacción con los turistas, se describen varios componentes inherentes a esta competencia que se han manifestado en el comportamiento de los dos estudiantes observados, al momento de dar sus discursos plurilingües. Se trata en primer lugar de la implementación específica del repertorio plurilingüe, y por otra parte del funcionamiento de la comunicación exolingue, tal como lo define Porquier (1974).

\section{- Realización de la compe- tencia plurilingüe en la co- municación}

Durante el intercambio con los turistas, los dos estudiantes observados implementaron efectivamente una competencia pluri- lingüe mediante la utilización de su repertorio verbal trilingüe, de manera diferenciada.

En primer lugar, combinaron las diferentes lenguas disponibles en su repertorio, poniendo en práctica una distribución funcional complementaria , utilizando sistemáticamente las lenguas "meta" para efectuar la intención comunicativa principal que consistía en explicar el programa turístico, y la lengua materna para responder a las preguntas de los turistas.

Por otra parte, se puede observar que las competencias implementadas fueron claramente parciales. Los dos estudiantes se "limitaron" a realizar una actividad lingüístico-comunicativa muy precisa, de tipo interaccional (guía-turistas), que sólo movilizó las aptitudes de expresión y de comprensión oral inscritas en una situación comunicativa muy particular.

\section{El funcionamiento de la comunicación exo-y plurilingüe}

\section{El carácter exolingue de la comunicación plurilingüe}

El carácter claramente plurilingüe de esta conversación exolingue se visibilizó por varias manifestaciones de contacto entre las lenguas, como por ejemplo la creación de articulaciones originales entre las lenguas en cuestión, e incluso la creación de nuevas for- 
mas lingüísticas idiosincrásicas e híbridas que comprueban la existencia de interconexiones entre los microsistemas de las diferentes lenguas en juego. Estas secuencias e intercambios bilingües aparecieron con más frecuencia entre segmentos lexicales o gramaticales de L1 y L2, y eso probablemente, por las representaciones de parentesco entre lenguas como el francés y el español.

\section{Marcas de contacto entre las lenguas: el polo de "fusión" y el polo de "distinción"}

Se pueden agrupar las manifestaciones concretas de las marcas de contacto entre las lenguas, las llamadas "marcas transcódicas", recogiendo los conceptos de alternancia de código, préstamo, interferencia (Ludi, 1987), alrededor de dos polos: el polo de "fusión" representando las formas que aproximan los dos sistemas lingüísticos por la generación de cierta reestructuración en uno de ellos, a raíz de un préstamo de la otra lengua, por ejemplo el uso en francés del adverbio "premier" reestructurando la estructura existente "en premier lieu", y aproximándolo del español "primero". Por otra parte, el polo de "distinción" agrupa las marcas que se definen por el respeto de las propiedades respectivas de cada sistema lingüístico (Py, 1992), como la alternancia propiamente dicha.

En el contexto de los dos estudiantes, los casos de alternancia de código de "distinción", deriva- dos del uso autorizado de la lengua materna, se presentaron de una manera más frecuente que los casos de "fusión", los cuales se concretizaron en préstamos o interferencias y que revelan una competencia plurilingüe más evolucionada. En este sentido, se ha podido observar que el estudiante con buen desempeño se sirvió más de la alternancia de código que la otra estudiante, y que también recurrió más a la herramienta de la "fusión", por lo que se hace acreedor de una competencia plurilingüe más evolucionada, debido podría decirse a su mayor tolerancia hacia las variaciones. En resumen, se ha identificado una presencia más importante de estrategias plurilingües y de manifestaciones de contacto entre las lenguas.

En cuanto a los pares de lengua implicadas en esta alternancia, la transferencia de una lengua parece claramente influenciada por la distancia lingüística entre una y otra, lo que permitiría explicar la frecuencia más grande de casos de alternancia entre francés y español. Esto nos permite establecer como hipótesis que la proximidad o el parentesco entre las lenguas podría ser efectivamente un factor importante a tomar en cuenta en la explicación de la frecuencia del fenómeno de la alternancia de código. Por otra parte, se ha podido observar en los dos estudiantes una tendencia de "compartimentar" las lenguas "meta" (el francés y el inglés), en el sentido en que se han producido muy pocos casos de in- terferencia entre estas lenguas.

\section{Conclusiones}

En conclusión se puede considerar que, a pesar de una implementación diferenciada de la competencia plurilingüe y una cualidad de desempeño diferente en los dos estudiantes observados, al parecer, el enfoque accional aplicado en un proyecto de clase trilingüe ha producido sus resultados, en el sentido en que un estudiante principiante que dispone de instrumentos lingüísticos limitados, puede llegar a comunicar con interlocutores extranjeros en diferentes lenguas, utilizando al máximo todas sus variedades lingüísticas en su repertorio plurilingüe, y utilizando los recursos de la comunicación exolingue.

De manera general, parece claro que a través de la movilización de competencias en varias lenguas y culturas, este enfoque puede perfectamente ponerse al servicio de un contexto educativo específico hondureño. Por una parte, en las situaciones de enseñanza de inglés en el sistema secundario, creando más posibilidades de "cohabitación" con la segunda lengua extranjera, el francés, y las otras lenguas extranjeras en ascenso".

Por otra parte, en el campo de la educación intercultural bilingüe se puede trabajar con las lenguas minoritarias para ir promoviendo una enseñanza del español en binomio con la lengua indígena en cuestión. En fin, esta propuesta de cohabitación de lenguas y culturas, 
constituye sin duda una metodología novedosa, que puede convertirse en un gran apoyo al sistema educativo nacional.

\section{Referencias}

Borg, S. (2001). Perspective actioonnelle dans le Cadre Commun Européen de Référence [Perspectiva accional en el Marco Común Europeo de Referencia]. Synergies Brésil, 61-72.

Coste, D., Moore, D., Zarate, G. (1998). Compétence plurilingue et pluriculturelle [Competencia Pluriligue y Pluricultural].Le Français dans le Monde Recherches et Applications. Apprentissage et usage des langues dans le cadre européen, 8-67.

Escuela de Altos Estudios de

Hostelería y Turismo. (2006). Vacaciones en América Central, Módulos de Francés para profesionales del Turismo. San José, Costa Rica: Ediciones Perro Azul

Conseil de l'Europe.(2001). Marco Común Europeo de Referencia. Strasbourg, Francia: Division des Langues Vivantes.

Fergusson, C.F. (1959).Diglossia

[Diglosia].Word 15.

Fishman, J.(1970).Sociolinguistics: a brief introduction [Sociolingüística :Una breve introducción]. Rowley, Estados Unidos: Newbury House.

Gremmo, M. (1997). Matériaux d'enseignement et apprentissage de langue : les options didactiques des modules de français pour les professionnels du tourisme [Insumos para la enseñanza y aprendizaje de lenguas : Las opciones didácticas de los módulos de francés para los profesionales de turismo]. Mélanges Pédagogiques n. 23. Université de Nancy, Francia.

Gumperz, J. (1962). Types of linguistic communities [Tipos de comunidades linguisticas]. Anthropological linguistics, 4, 1.

Ludi, G. (1987).Devenir bilingue, parler bilingue [Hacerse bilingüe, hablar bilingüe].Tübingen, Suiza: Niemeyer.

Porquier, R. (1984).Communication exolingue et apprentissage des langues [Comunicación exolingue y aprendizaje de lenguas].Acquisition d'une langue étrangère. Université Paris-VIII et Université de Neuchâtel, Francia.

Py, B.(1992).Regards croisés sur les discours du bilingue et de l'apprenant ou le retour sur le rôle de la langue maternelle dans l'acquisition de la langue seconde [Perspectiva entrecruzada sobre los discursos del bilingüe y del aprendiz, o reconsideración del rol de la lengua materna en la adquisición de la lengua segunda].Autour du multilinguisme (Lidil.No.6 juin, pp.10-25). Grenoble. Francia

Richterich, R., Chancerel, J. (1977). L'identification des besoins des adultes apprenant une langue étrangère [La iden- tificación de las necesidades de los adultos en el aprendizaje de un idioma extranjero ]. Conseil de l'Europe. Strasbourg, Francia. 